\title{
Corrigendum: Inhibition of Lung Tumor Development in ApoE Knockout Mice via Enhancement of TREM-1 Dependent NK Cell Cytotoxicity
}

\author{
Yong Sun Lee, In Jun Yeo, Ki Cheon Kim, Sang-Bae Han and Jin Tae Hong* \\ College of Pharmacy and Medical Research Center, Chungbuk National University, Cheongju, South Korea
}

OPEN ACCESS

Edited and reviewed by: Katy Rezvani,

University of Texas MD Anderson

Cancer Center, United States

*Correspondence: Jin Tae Hong

jinthong@chungbuk.ac.kr

Specialty section: This article was submitted to

Cancer Immunity

and Immunotherapy,

a section of the journal

Frontiers in Immunology

Received: 21 December 2021 Accepted: 31 December 2021 Published: 21 January 2022

Citation:

Lee YS, Yeo IJ, Kim KC,

Han S-B and Hong JT (2022) Corrigendum: Inhibition of Lung Tumor Development

in ApoE Knockout Mice via Enhancement of TREM-1 Dependent NK Cell Cytotoxicity.

Front. Immunol. 12:840856. doi: 10.3389/fimmu.2021.840856
Keywords: Lung tumor development, apolipoprotein E, TREM-1, T-bet, NK cells

\section{A Corrigendum on}

Inhibition of Lung Tumor Development in ApoE Knockout Mice via Enhancement of TREM-1 Dependent NK Cell Cytotoxicity

by Lee YS, Yeo IJ, Kim KC, Han S-B and Hong JT (2019). Front. Immunol. 10:1379. doi: 10.3389/fimmu.2019.01379

In the original article, there was a mistake in Figure 3C as published. In Figure 3C, overlapped images were mistakenly used. For reliability, the experiment (transwell migration assay) was performed again, and the description of the experimental method was revised. The corrected Figure 3 with a legend and a revised method appear below.

The authors apologize for this error and state that this does not change the scientific conclusions of the article in any way. The original article has been updated.

\section{TRANSWELL MIGRATION ASSAY}

Serum-starved B16F10 cells $\left(1 \times 10^{5}\right.$ cells in $100 \mu$ serum-free medium) were added to the collagen pre-coated transwell insert (pore size $8.0 \mu \mathrm{m}$; distance $6.5 \mathrm{~mm}$ ), and the culture well was filled with $600 \mu \mathrm{l}$ of the complete medium containing 10\% FBS. After $18 \mathrm{~h}$, cells were fixed with $70 \%$ ethanol for $10 \mathrm{~min}$ and stained with crystal violet solution. Then, non-migrated cells in the upper side of 


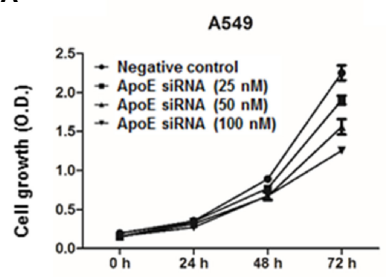

$\mathrm{NCl}-\mathrm{H} 460$

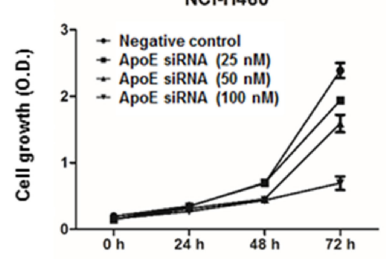

B16F10

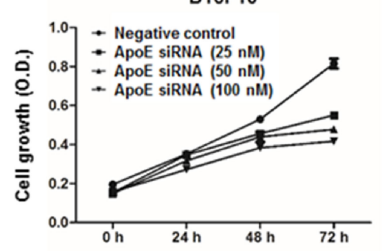

B

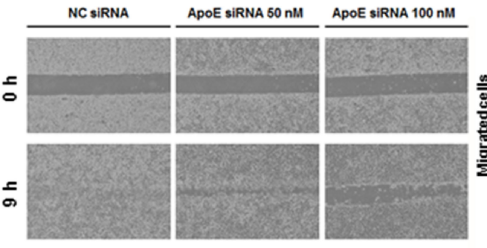

C
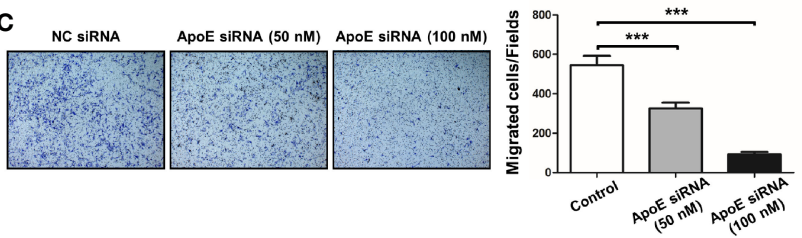

D

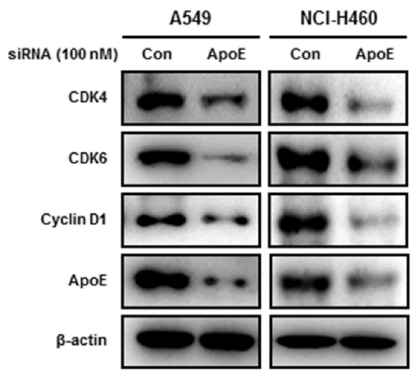

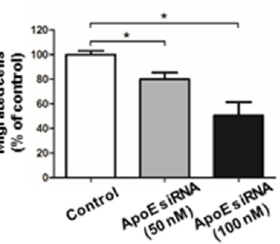
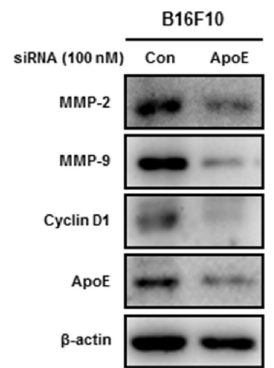

FIGURE 3 | Effect of ApoE knockdown on cell growth and migration in cancer cells. (A) Lung cancer cells (A549 and NCl-H460) and B16F10 cells were plated on 96-well plates $\left(1 \times 10^{3}\right.$ cells per well) and transfected with the negative control (NC) siRNA or ApoE siRNA (25, 50 or $\left.100 \mathrm{nM}\right)$ for indicated time points. Cell growth was measured by MTT assay $(n=5)$. (B) B16F10 cells were seeded on $\mu$-Slide and transfected with NC siRNA or ApoE siRNA (50 or 100 nM). Cells were cultured to confluent on $\mu$-Slide $(n=3)$. After silicon-wall removal, cells were allowed to migrate into cell-free zone. Cell migration was detected at various times post-silicon-wall removal by a microscopy at 100X. (C) B16F10 cells transfected with NC siRNA or ApoE siRNA (50 or 100 nM) were seeded onto transwell inserts pre-coated with collagen on the bottom side and loaded into culture well filled with growth medium containing 10\% FBS as a chemoattractant ( $n=3)$. After $18 \mathrm{~h}$ incubation, transwell inserts were fixed and stained by crystal violet solution. Bar graphs represent cell-migration distance or number of migrated cells. ${ }^{*} p<0.05$ and ${ }^{* \star *} p<0.0001$. (D) Cells were transfected with NC siRNA or ApoE siRNA (100 nM) for $24 \mathrm{~h}$. Cell extracts were analyzed by Western blotting. Samples (30 $\mu \mathrm{g})$ were resolved on SDSPAGE and detected with specific antibodies against CDK4, CDK6, Cyclin D1, MMP-2, MMP-9 and ApoE. $\beta$-actin was used as a loading control.

transwell insert were removed using a cotton swab. Three random fields of each insert were counted and photographed under a light microscope (x100, Olympus, Tokyo, Japan).

Conflict of Interest: The authors declare that the research was conducted in the absence of any commercial or financial relationships that could be construed as a potential conflict of interest.

Publisher's Note: All claims expressed in this article are solely those of the authors and do not necessarily represent those of their affiliated organizations, or those of the publisher, the editors and the reviewers. Any product that may be evaluated in this article, or claim that may be made by its manufacturer, is not guaranteed or endorsed by the publisher.

Copyright $\odot 2022$ Lee, Yeo, Kim, Han and Hong. This is an open-access article distributed under the terms of the Creative Commons Attribution License (CC BY). The use, distribution or reproduction in other forums is permitted, provided the original author(s) and the copyright owner(s) are credited and that the original publication in this journal is cited, in accordance with accepted academic practice. No use, distribution or reproduction is permitted which does not comply with these terms. 